Document downloaded from:

http://hdl.handle.net/10251/35101

This paper must be cited as:

Luis Guijarro; Vicent Pla; Bruno Tuffin; Patrick Maillé; Vidal Catalá, JR.; Guijarro (2011). Competition and bargaining in wireless networks with spectrum leasing. IEEE GLOBECOM 2011. Institute of Electrical and Electronics Engineers (IEEE). 1-6. doi:10.1109/GLOCOM.2011.6133605.

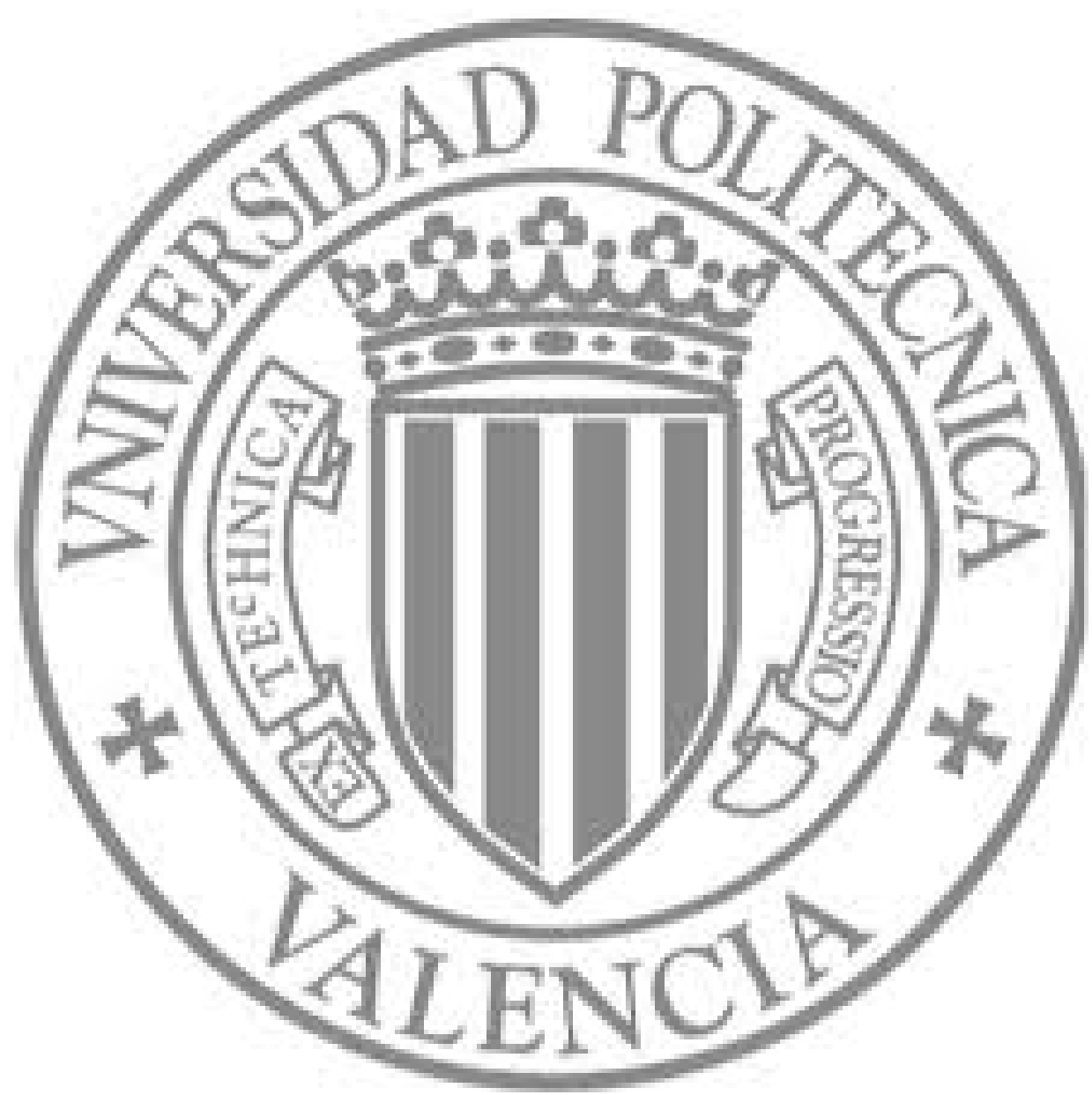

The final publication is available at

http://dx.doi.org/10.1109/GLOCOM.2011.6133605

Copyright Institute of Electrical and Electronics Engineers (IEEE) 


\title{
Competition and bargaining in wireless networks with spectrum leasing
}

\author{
Luis Guijarro*, Vicent Pla*, Bruno Tuffin ${ }^{\dagger}$, Patrick Maillé $\ddagger$ and Jose R. Vidal ${ }^{*}$ \\ *Universitat Politècnica de València, Spain \\ $\dagger$ INRIA Rennes, France \\ $\ddagger$ Telecom Bretagne, France
}

\begin{abstract}
The case for a competitive market operated by a Mobile Network Operator (MNO) and a Mobile Virtual Network Operator (MVNO) is analysed in the paper. The resource that is leased by the MNO to the MVNO is spectrum. The MNO and the MVNO compete à la Bertrand posting subscription prices and the mobile users may choose to subscribe to one operator. The scenario is modeled by a three-level game comprising a bargaining game, which models the spectrum leasing by the MNO; a competition game, which models the price competition between the MNO and the MVNO; and a subscription game, which models the subscription choice by the mobile users, and the outcome of which may be either not to subscribe, to subscribe to the MNO or to subscribe to the MVNO. The game is solved through backward induction, and each level has a specific solution concept: Shapley value, for the bargain; Nash equilibrium, for the competition; and Wardrop equilibrium, for the subscription. The paper assesses which conditions lead to an equilibrium where the competition does take place, which are expressed as restrictions for the spectrum leasing price agreed at the bargaining, and the spectrum efficiency improvement achieved by the MVNO. Furthermore, it argues that the amount of the leased spectrum should be fixed exogenously in order to achieve optimal user and social welfares.
\end{abstract}

\section{INTRODUCTION}

Cellular wireless networks are managed by operators which have bought a license, giving them the exclusive right to use a fixed part of the radio spectrum for their customers. Regulatory rules initially prevented them to resale a part of their license rights. This fixed distribution of the spectrum has been criticized [1]. Spectrum sharing has been implemented in many countries as the simplest way to allow a new potential mobile operator to access the market. But the viability of such a system has to be precisely studied. It has for example been observed in practice that the MVNO integration has not always been successful in different countries [2]: it appears that horizontally structured markets (i.e., with several independent participants contributing to the different levels of service providing) offer greater possibilities for MVNO profitability than markets where a vertically integrated incumbent controls most of the supply chain.

In this paper, we focus on the interaction between an MNO and an MVNO. More precisely, the paper deals with

This work has been supported by Euro-NF Network of Excellence for all authors, the Spanish Government through projects TIN2010-21378-C02-02 and TIN2008-06739-C04-02 for the Spanish authors and the French research agency through the CAPTURES project for the French authors. primary-secondary sharing [3]. In this setting, the MNO is a Primary Operator (PO), having acquired a license which gives it the right to use spectrum, while the MVNO is a Secondary Operator (SO), which leases a fraction of the spectrum licensed to the PO. The $\mathrm{SO}$ is assumed to have deployed a new technology which allows a more efficient use of the spectrum than the one used by the PO. While authorizing the SO to use the PO's spectrum will permit the PO to get additional revenue from leasing its spectrum, it may also induce losses due to customers leaving to the newcomer. Increasing the leasing price may limit PO's losses but it may also then prevent the SO from getting any profits, and therefore from entering the market. As a result, it is not obvious whether the PO will let the SO use its spectrum without any regulatory intervention. In particular, the price that the PO will charge the SO has to be carefully chosen to optimize resource usage while ensuring sustainability of the PO-SO association [4]. As the operators have conflicting interests, we consider the modeling and analysis framework of non-cooperative game theory [5], which studies the interactions between selfish actors (also named players).

Our contribution is the introduction and analysis of a model representing the economic interactions between the $\mathrm{PO}$, the $\mathrm{SO}$, and the users, and in general investigating the viability of such a system. We design a three-level game where at the highest level the operators agree on the amount of leased spectrum and the corresponding unit price; at the intermediate level, the operators play on the price they will propose to users; and at the lowest level users distribute themselves between operators depending on price and QoS.

Pricing telecommunication services in general has been the topic of an extensive literature. Most works look at a monopolist provider and very few take care of the competition for customers. When it comes to competition and secondary usage, cognitive networks or MVNOs, the literature is to our knowledge even more limited; see for instance [6], [7]. Competition is taken into account in [8]-[10], although through demand functions that include indirect effects. By contrast, we explicitly model user utilities and behavior to deduce demands. The closest related works are to our knowledge [11] and [12]. In those papers, the competition between the MNO and the MVNO is also analyzed, but they differ from our work in two aspects: first, congestion is measured by the response 


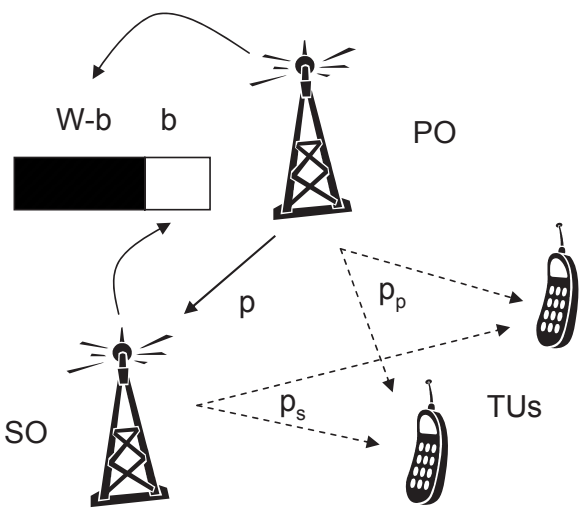

Fig. 1. Scenario.

time at a simple $\mathrm{M} / \mathrm{M} / 1$ queue, while we consider here a measure more representative of wireless networks. Second, the higher-level interactions are based on contract setting, while we consider here simple fixed-capacity, fixed-lease contracts but focus on negotiations for revenue sharing. The paper also improves on a previous publication [13], where a simpler model was developed for analyzing the same scenario as here. Actually, we here extend the analysis in two significant ways. First, it models the bargaining between the operators over the price of the leased spectrum. And second, it models the user willingness to pay, while the previous publication did not, so that users were unable to choose to subscribe to neither the PO nor the SO.

The rest of this paper is organized as follows. Next section describes the scenario under analysis and develops the model, specifying each one of the three phases that comprise the game. Section III evaluates the different competitive equilibria that may arise in the game, discussing the effect of the different parameters on the game outcome and assessing the outcome from the point of view of the welfare. And finally, Section IV draws some conclusions.

\section{MODEL}

The basic model we will analyze is depicted in Fig. 1. It is made of one PO and one SO which compete for the provision to the users; and (tertiary) users that are assumed to have dual terminals, so that no technology-related switching costs are incurred when making the subscription decision. The PO leases an amount of $b \mathrm{MHz}$ to the SO, keeping for itself the rest, up to the total amount of its licensed $W \mathrm{MHz}$. The SO pays for that amount a price $p$ m.u. ${ }^{1}$ per MHz. Users pay a subscription fee to the operator they have decided to subscribe to. A user would pay $p_{p}$ m.u. if she subscribed to PO service, or $p_{s}$ m.u. if she subscribed to SO service. All three prices $p$, $p_{p}$ and $p_{s}$ are referred to the same time period.

We assume that the operators compete à la Bertrand [5], that is, they are playing a one-shot simultaneous game where

${ }^{1}$ m.u. $=$ monetary units.
PO and SO strategies are $p_{p}$ and $p_{s}$, respectively. Unlike the model presented in [13], where both the price $p$ and the leased spectrum amount $b$ were exogenously determined, in the model described below the leasing price $p$ is the result of a bargaining process between the PO and the SO. Finally, each user will subscribe to the service providing the highest utility, which will be shown to depend on the quality of service and on the price. Assuming that the number of users $(n)$ is high enough, the individual subscription decision of each user will not affect the utility perceived by the rest. Then, the equilibrium notion is the so-called Wardrop equilibrium [14] [15], where users are indifferent between choosing one operator or the other one, and therefore no user has an incentive to switch. Unlike the model presented in [13], users may choose not to subscribe to any service. This issue will be discussed below.

The strategic interaction between the two operators and the $n$ users is modeled as a three-level multi leader-follower game. A standard way to analyze this sort of games is by means of backward induction: the game at the second phase is played knowing - anticipating - what would be the outcome of the game at the third phase; similarly, the game at the first phase is played anticipating the outcome of the game at the second phase. We now show how the outcomes of those games can be computed.

\section{A. Subscription game-third phase}

In this phase, a pair of values $b$ and $p$ has been agreed and the prices $p_{p}$ and $p_{s}$ have been announced.

The utility that the users receive from each operator depends on three factors:

Basic willingness: following [16], we assume that the users are heterogeneous in their basic willingness to pay for the service, but homogeneous in their valuation of the quality of the service. Specifically, we model the basic willingness as a random variable $\tau$ with a complementary distribution function which is exponentially decreasing ${ }^{2}$ :

$$
F_{\tau}^{c}(x) \triangleq P(\tau>x)= \begin{cases}1 & \text { if } x<0 \\ e^{-a x} & \text { if } x \geq 0\end{cases}
$$

where $a$ is a parameter which denotes the decreasing gradient for the complementary distribution function: the larger $a$, the more steeply the function decays.

Quality of service: each operator exploits, during each subscription period, an amount of spectrum which is agreed at the end of the first phase: $b$ for the $\mathrm{SO}$ and $W-b$ for the PO. Furthermore, each operator is assumed to use a different technology, which results in different levels of spectral efficiency: $k^{(p)}$ and $k^{(s)}$ are the spectral efficiencies for the $\mathrm{PO}$ and the SO, respectively. Both the modulation schemes and the medium access control mechanisms are modeled by the numbers $k^{(p)}$ and $k^{(s)}$. We assume that $k^{(p)}<k^{(s)}$, i.e. the SO uses a more efficient technology than the PO. The product of the spectrum by the spectral efficiency

\footnotetext{
${ }^{2}$ This distribution allows for a simpler analytical processing than the more common uniform distribution.
} 
for each operator, divided by the number of users which subscribe to it, will give the transfer rate that is offered to each user [17]. We propose to use this transfer rate as the quality factor $\left(Q_{p}\right.$ and $\left.Q_{s}\right)$ contributing to the user utility, through an increasing concave (logarithmic) function. Specifically, $Q_{p}=\log \left(k^{(p)}(W-b) / n_{p}\right), Q_{s}=\log \left(k^{(s)} b / n_{s}\right)$, where $n_{p}$ (resp., $n_{s}$ ) is the number of users subscribing to the PO-resp., the SO.

Price: the higher the subscription price, the lower the user utility. We consider quasi-linear user utility functions: the utility is the difference between the value of the service and the price paid.

Consider a user with willingness to pay $\tau$. Based on the above discussion, the expressions for that user utility if subscribing to the PO or the SO are, respectively, $U_{p}=\tau+Q_{p}-p_{p}$ and $U_{s}=\tau+Q_{s}-p_{s}$. A selfish user will subscribe to the service provided by the PO rather than the SO if $U_{p}>U_{s}$, and reciprocally.

As stated above, at a user (Wardrop) equilibrium, the $n_{p}^{*}+n_{s}^{*}$ users which subscribe to a service should spread between the PO and the SO such that no user has an incentive to switch to the competing operator. For this to happen, $U_{p}=U_{s}$ must hold for every subscriber, or equivalently $Q_{p}-p_{p}=Q_{s}-p_{s}$. Let $\alpha$ denote the fraction of subscribers that subscribe to the $\mathrm{PO}$, and $1-\alpha$, to the SO.

Additionally, only those $n_{p}^{*}+n_{s}^{*}$ users which have a basic willingness high enough so as to obtain a positive utility will subscribe to the service. We now introduce the fraction $\beta \triangleq$ $\left(n_{p}^{*}+n_{s}^{*}\right) / n$ of users that subscribe to the service. After some manipulations, the following expression can be obtained

$$
\beta= \begin{cases}1 & \text { if } p_{p} \leq \hat{p}_{p}, \\ \left(\frac{k^{(p)}(W-b)}{n \alpha} e^{-p_{p}}\right)^{\frac{a}{a+1}} & \text { if } p_{p} \leq \hat{p}_{p}\end{cases}
$$

where $\hat{p}_{p}=\log \left(k^{(p)}(W-b) /(n \alpha)\right)$. The interested reader is referred to [18] for the detailed derivation of the expression. Then $n_{p}$ and $n_{s}$ can be expressed as functions of $\beta$ and $\alpha$ as $n_{p}=\alpha \beta n$ and $n_{s}=(1-\alpha) \beta n$.

\section{B. Price competition game-second phase}

In this phase, a pair of values $b$ and $p$ has been agreed on, and each operator chooses its pricing strategy so as to maximize its profits. The outcome of the subscription game is assumed to be anticipated by both operators, and taken into account in the pricing decisions.

The profits of the PO and the SO can be expressed, respectively, as $\Pi_{p}=n_{p} \cdot p_{p}+p \cdot b-C_{p}$ and $\Pi_{s}=n_{s} \cdot p_{s}-p \cdot b-C_{s}$. where $C_{p}$ and $C_{s}$ are the management-and infrastructure for the PO- costs born by the PO and the SO, respectively.

When solving the equilibrium equations for the second and the third phase, $n_{p}$ and $n_{s}$ may be expressed as functions of $p_{p}$ and $p_{s}$, so that operator profits are functions of $p_{p}$ and $p_{s}$ only: $\Pi_{p}=\Pi_{p}\left(p_{p}, p_{s}\right), \Pi_{s}=\Pi_{s}\left(p_{p}, p_{s}\right)$.

Now, turning our attention to the pricing game, the equilibrium strategies $p_{p}^{*}$ and $p_{s}^{*}$ are given by the Nash equilibrium conditions [5]: $\Pi_{p}\left(p_{p}^{*}, p_{s}^{*}\right) \geq \Pi_{p}\left(p_{p}, p_{s}^{*}\right), \quad \forall p_{p} ; \Pi_{s}\left(p_{p}^{*}, p_{s}^{*}\right) \geq$ $\Pi_{s}\left(p_{p}^{*}, p_{s}\right), \quad \forall p_{s}$; meaning that no operator can unilaterally increase its profits by a price change.

Under the assumption that the partial derivatives of $\Pi_{p}$ and of $\Pi_{s}$ with respect to $p_{p}$ and $p_{s}$ exist, the Nash equilibrium can be determined by looking at the solutions of the firstorder conditions. After some algebra, the following equation is obtained for $\alpha^{*}$, the fraction of subscribers selecting the PO at the equilibrium:

$$
\begin{aligned}
\log \alpha^{*}+ & \frac{a+1}{a+1-\alpha^{*}}=\log \left(1-\alpha^{*}\right) \\
& +\frac{a+1}{a+1-\left(1-\alpha^{*}\right)}+\log \left(\frac{k^{(p)}(W-b)}{k^{(s)} b}\right) .
\end{aligned}
$$

Note that the function $f(x)=\log x+\frac{a+1}{a+1-x}$ is continuous, increasing for $x>0$ and that $\lim _{x \rightarrow 0}=-\infty$. We can then infer that there exists a unique value of $\alpha \in(0,1)$ which satisfies (3).

\section{Operators bargaining-first phase}

As stated at the beginning of this section, the price $p$ and the amount of spectrum $b$ are subject to a bargaining process between the PO and the SO, which is conducted before the subscription prices are advertised by the operators and the subscription decision is performed by the users.

Following [19], we model the bargaining as a noncooperative game where the incumbent operator (the PO) has full bargaining power and therefore offers a take-it-orleave-it offer to the entrant operator (the SO). For the sake of simplicity, we stand by the full bargaining case, although alternative assumptions are possible, as discussed later.

The game is analyzed as a dynamic game in an extensive form. Following backward induction, depending on the values of $(b, p)$ which characterize the PO offer, the bargaining outcome is characterized as follows:

1) The PO will make an offer only if it prefers the competition outcome compared to the monopolistic outcome, that is, $\Pi_{p} \geq \Pi_{m}$.

2) If the offer made by the PO induces $\Pi_{s} \geq 0$, the SO will accept the offer. Otherwise, $\Pi_{s}<0$, and the SO will refuse it.

To compute $\Pi_{m}$, the problem should be stated as an optimal decision problem, such that the optimal price $p_{m}^{*}$ should fulfill $\Pi_{m}\left(p_{m}^{*}\right) \geq \Pi_{m}\left(p_{m}\right), \forall p_{m}$. Then we obtain $p_{m}^{*}=1+1 / a$ and

$$
\Pi_{m}\left(p_{m}^{*}\right)=\frac{n}{e}\left(1+\frac{1}{a}\right)\left(\frac{k^{(p)} W}{n}\right)^{\frac{a}{a+1}}-C_{p} .
$$

We now proceed to define the feasibility region of values $(b, p)$ which allow for a competitive equilibrium to result. From the condition $\Pi_{s} \geq 0$, we derive:

$$
p \leq U(b) \triangleq \frac{n\left(1-\alpha^{*}\right) p_{s}^{*}}{b}\left(\frac{k^{(p)}(W-b)}{n \alpha^{*}} e^{-p_{p}^{*}}\right)^{\frac{a}{a+1}}-\frac{C_{s}}{b} .
$$


From the condition $\Pi_{p} \geq \Pi_{m}$, we get:

$$
\begin{aligned}
& p \geq L(b) \triangleq \frac{n}{b}\left(\frac{k^{(p)} W}{n} e^{c}\right)^{\frac{a}{a+1}} \\
& \cdot\left(\left(1+\frac{1}{a}\right) e^{-1}-\left(\left(1-\frac{b}{W}\right) \frac{1}{\alpha^{*}} e^{-p_{p}^{*}}\right)^{\frac{a}{a+1}} \alpha^{*} p_{p}^{*}\right) .
\end{aligned}
$$

Again the detailed derivation of the expressions may be found at [18]. Therefore, a non-empty feasibility region will exist if $L(b) \leq U(b)$ and a point $(b, p)$ will be in the feasibility region iff

$$
\max (0, L(b)) \leq p \leq U(b)
$$

The final bargaining outcome will depend on the specific assumptions made over the bargaining process. If the incumbent has full bargaining power, it will ask for a profit-maximizing price $p$, i.e. such that the equality holds in (5), $p=U(b)$. Given that $\Pi_{p}$ is monotonically increasing on the value $p$, this would provide the incumbent with maximum profits.

Apart from the full bargaining power case, other solution concepts can be borrowed from the cooperative theory for choosing the value of $p$. By noting that $\left.\Pi_{p}\right|_{p=L(b)}=\Pi_{m}$ and $\left.\Pi_{s}\right|_{p=U(b)}=0$, the expressions for $\Pi_{p}$ and $\Pi_{s}$ can be rewritten as $\Pi_{p}=\Pi_{m}+(p-L(b)) b$ and $\Pi_{s}=U(b)-p$, from what it follows that $\Pi_{p}+\Pi_{s}=\Pi_{m}+\Delta$, where $\Delta=(U(b)-L(b)) b \geq 0$ is the amount by which the total profit is incremented if the SO enters the market. Note that $\Delta$ does not depend on $p$.

We have then transformed the problem of setting the price $p$ at which the $\mathrm{PO}$ sells bandwidth to the SO, to an equivalent one of deciding how the extra profit $\Delta$ is shared between the PO and the SO. For this equivalent problem, the Shapley value [20] provides a fair allocation of the payoff obtained by the PO-SO coalition, such that each operator (PO or SO) will receive a share of the profits proportional to its contribution to the total profits. More precisely, each operator will receive the profits it would get if alone and half of the profits increase that its presence brings to the total profits in case of a coalition. In our case, the Shapley value allocation yields

$$
\begin{aligned}
& \Pi_{p}=\frac{1}{2} \Pi_{m}+\frac{1}{2}\left(\Pi_{m}+\Delta-0\right)=\Pi_{m}+\frac{\Delta}{2}, \\
& \Pi_{s}=\frac{1}{2} 0+\frac{1}{2}\left(\Pi_{m}+\Delta-\Pi_{m}\right)=\frac{\Delta}{2},
\end{aligned}
$$

which correspond to

$$
p=\frac{U(b)+L(b)}{2} .
$$

Alternatively, the problem of agreeing a value for $p$ can be casted into a two person bargaining problem in which the disagreement point is $\left(\Pi_{m}, 0\right)$ and the players' strategies are their offers about $p$. In this setting, both the Nash bargaining solution and the Kalai-Smorodinsky bargaining solution [20] can be computed and it can be shown that they yield the same results as the provided by the Shapley value (see (8) and (9)).
TABLE I

DEFAULT PARAMETER SETTING

\begin{tabular}{c|c||c|c}
\hline Parameter & Value & Parameter & Value \\
\hline$n$ & $100000 \mathrm{users}$ & $k^{(p)}$ & $1 \mathrm{bit} / \mathrm{s} / \mathrm{Hz}$ \\
$W$ & $100 \mathrm{kHz}$ & $k^{(s)}$ & $1.2 \mathrm{bit} / \mathrm{s} / \mathrm{Hz}$ \\
$C_{p}$ & $10 \mathrm{u.m.}$ & $a$ & 1.7 \\
$C_{s}$ & $5 \mathrm{u} . \mathrm{m}$. & & \\
\hline
\end{tabular}

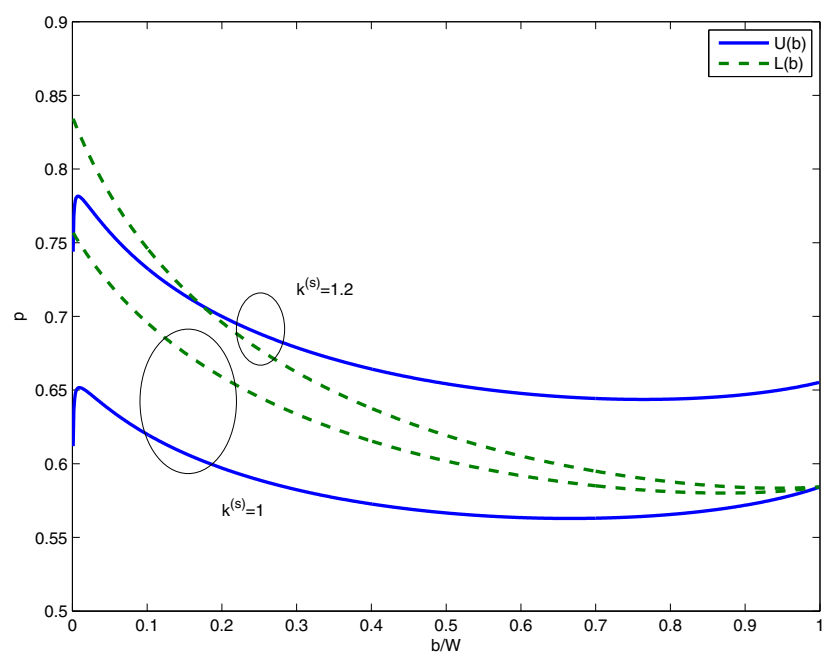

Fig. 2. Upper and lower limits for the feasible values of $p$.

Note, however, that the value $b$ is not determined by the bargaining, but only constrained by $L(b) \leq U(b)$. As shown in the next section, welfare-either producer welfare, user welfare or social welfare-can determine the value $b$.

\section{RESULTS AND EQUILIBRIUM ANALYSIS}

In order to evaluate the different competitive equilibria, we propose to use the following indicators:

1) Operators profits $\Pi_{p}$ and $\Pi_{s}$.

2) User utilities, $U_{p}^{T}$ and $U_{s}^{T}$, computed as the expected values over those users that subscribe to the PO and to the $\mathrm{SO}$, respectively.

3) User welfare, $U W$, computed as the aggregated user utility over the total number of subscribers.

4) Social welfare, $S W$, computed as the sum of the user welfare and the producer welfare-i.e., $S W=U W+$ $\Pi_{p}+\Pi_{s}$.

We have conducted a series of numerical experiments in order to obtain a better understanding of the scenario from the point of view of the economic interactions. The values for the parameters, if not stated otherwise, are the ones shown in Table I.

\section{A. Feasible values for $(b, p)$}

The objective of this experiment is to characterize the feasibility region for $(b, p)$, that is, to find values $(b, p)$ where condition (7) is satisfied. Figure 2 shows the values of $U(b)$, given by (5), and $L(b)$, given by (6), as functions of $b$.

We see that for values of $b$ greater than a threshold value $b_{\text {min }}, L(b)<U(b)$ holds and, therefore, corresponding values 
for $p$ can be found such that the competition setting results in an equilibrium. More formally, a feasibility region $\mathcal{F}$ can be found such that

$$
\mathcal{F} \triangleq\left\{(b, p) \mid b_{\min } \leq b \leq b_{\max } \leq W, L(b) \leq p \leq U(p)\right\}
$$

where $b_{\text {min }}$ is such that $L\left(b_{\text {min }}\right)=U\left(b_{\text {min }}\right)$.

\section{B. Spectral efficiency}

We now assess the effect of $k^{(s)}$ on the feasibility region $(b, p)$. We have tested $1 \leq k^{(s)} \leq 1.2$. The case $k^{(s)}=1.2$ was shown in the previous section, and the case $k^{(s)}=1$ is shown in this section. Figure 2 shows again $U(b)$ and $L(b)$, as functions of $b$.

We see that, for the whole range of values of $b, L(b)>U(b)$ holds, so that there is no pair of values $(b, p)$ which results in a competitive equilibrium.

The result can be explained as follows. In the experiment, $k^{(s)}=k^{(p)}$, which means that the SO operator has not innovated in technology with respect to the PO operator. There is no incentive for either the PO or the SO to engage in a competition. Note that a similar conclusion has been achieved by [21], where the entry conditions of MVNOs are analyzed when incumbent MNOs exist: the latter do not have any incentive to lease network capacity unless the former chooses to provide differentiated services to the mobile users.

\section{On the value of the leased spectrum}

The objective of this experiment is to evaluate the effect of varying the amount of leased spectrum $b$. We assume that $p$ is agreed so that the Shapley value result for the profits sharing.

The following values are simultaneously represented as functions of $b$ on Fig. 3: $\alpha$ and $\beta, U_{p}^{T}$ and $U_{s}^{T}$, and $\Pi_{p}$ and $\Pi_{s}$, where the left $y$-axis is for $\alpha$ and $\beta$ and the right one is for $U_{p}^{T}$ and $U_{s}^{T}$. When $b<b_{\text {min }}$, meaning that it does not yield a competitive equilibrium, the represented values for the PO correspond to the monopolistic scenario.

We see that, with respect to the number of subscribers, throughout the interval $\left[0, b_{\min }\right)$, the PO remains as the monopolistic operator, i.e. $\alpha=1$ holds. When $b \geq b_{\text {min }}$, the SO operator enters the market, and the PO operator loses market share as $b$ increases, down to $\alpha=0$ when $b=W$. The number of subscribers in a competitive setting is always greater than in a monopolistic setting. Furthermore, $\beta$ reaches a maximum at an intermediate value $b_{\min }<b^{\prime}<W$.

With respect to profits $\Pi_{p}$ and $\Pi_{s}$, both operators increase their profits as the SO enters the market. Furthermore, they keep increasing as the SO operator gets more resources $(b>$ $b_{\text {min }}$ ) for providing service to its users.

With respect to utilities $U_{p}^{T}$ and $U_{s}^{T}$, the utility for the PO users does not change when the SO operator enters the market. Then, when competitive equilibrium is feasible, both utilities match $U_{p}^{T}=U_{s}^{T}$, and stay constant as $b$ increases. Actually, it can be shown that $U_{p}^{T}=U_{s}^{T}=1 / a$. When the results for both the number of subscribers $(\beta \cdot n)$ and the user utilities are jointly considered we can conclude that user welfare $(U W)$ reaches a maximum at the same value $b^{\prime}$ as $\beta$.

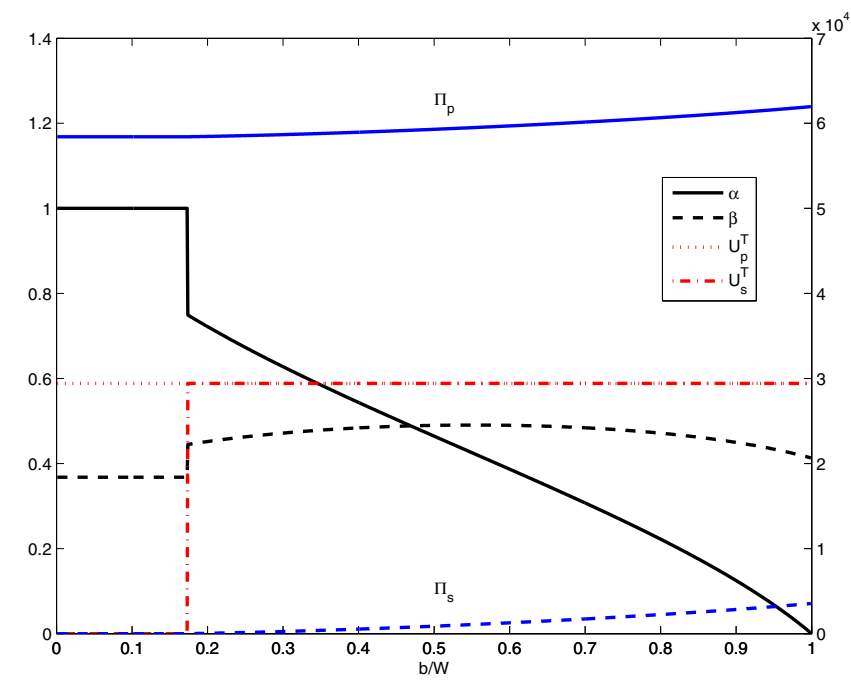

Fig. 3. Effect of variation of $b / W$

The above conclusions are valid regardless of the criteria used for fixing $p$. In this experiment, the value $p$ is obtained from (10). For any other value of $p$, specifically for $p=U(b)$-i.e. the incumbent has full bargaining power, the profits $\Pi_{p}$ and $\Pi_{s}$ would obviously be different. Nevertheless, the producer welfare, the equilibrium prices and the number of subscribers remain the same as those just represented and discussed, and so do consequently the user utility, the user welfare and the social welfare.

\section{Welfare-maximizing values for the leased spectrum}

Finally, we proceed to evaluate the optimum values $b$ of leased spectrum from the point of view of welfare. Specifically, we have computed and represented the following values in Fig. 4:

- Maximum value of $b / W$ in the feasibility region $\left(b_{\max } / W\right)$.

- Minimum value of $b / W$ in the feasibility region $\left(b_{\min } / W\right)$.

- Value of $b / W$ such that user welfare is maximized $\left(b_{U W} / W\right)$.

- Value of $b / W$ such that social welfare is maximized $\left(b_{S W} / W\right)$

We have performed different experiments varying $a$, which is the parameter of the basic user willingness (see Eq. (1)).

We see that, in all cases, the value $b / W$ can reach the value 1 , which is the case where the whole spectrum $W$ is leased by the SO. In other words, $b_{\max }=W$ in (11). As regards $b_{\text {min }} / W$, as $a$ increases, $b_{\text {min }}$ tends to zero, which would imply that even a small amount of leased spectrum would be profitable for the PO and the SO.

As regards the user welfare and the social welfare, we can identify five different zones in the graph, depending on the value of parameter $a$ :

- In the first zone, both $b_{U W}$ and $b_{S W}$ equal $b_{\text {min }}$, which means that the optimum for the users and for the whole 


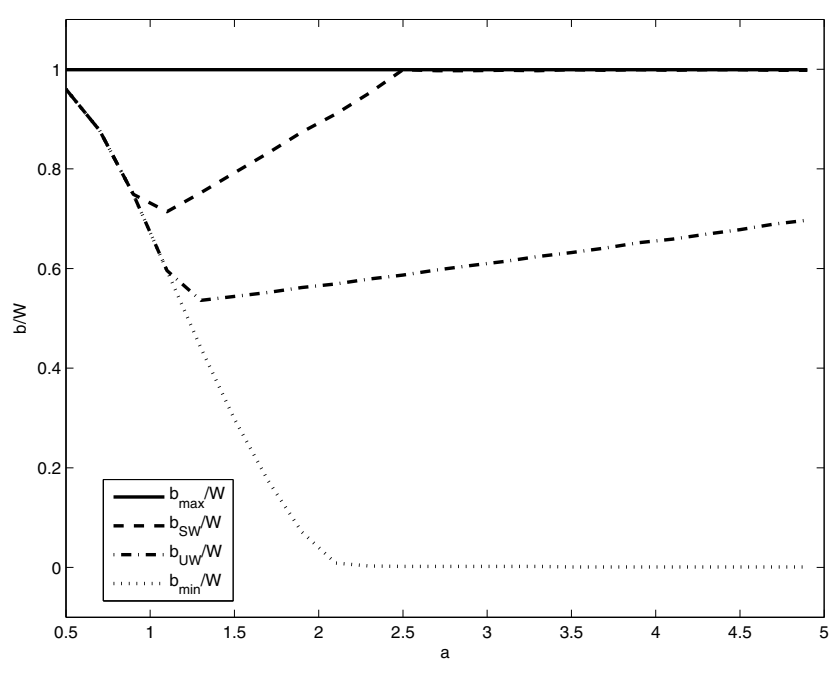

Fig. 4. Leased spectrum values which yield maximum user welfare and maximum social welfare

is that the PO leases the minimum amount of spectrum so that the entry is profitable for both PO and SO.

- In the second zone, $b_{U W}$ equals $b_{\min }$ but $b_{S W}$ detaches itself from $b_{m i n}$, which means that the optimum for the whole is now $b_{\min }<b_{S W}<b_{\max }$.

- In the third zone, $b_{U W}$ detaches itself from $b_{\text {min }}$, which means that the optima for the users and for the whole are intermediate values between $b_{\min }$ and $b_{\max }$.

- In the fourth zone, $b_{S W}$ reaches $b_{\max }=W$, so that the optimum for the whole system is that the PO leases the total amount of spectrum to the SO, whereas the optimum for the users is not to lease that much.

- In the fifth zone, not shown in the graph, $b_{U W}$ also reaches $b_{\max }=W$, so that the optimum for the users is also that the PO leases the whole amount of spectrum to the SO.

The above results mean that the degenerate case $b / W=1$, which is the optimum from the point of view of the producer welfare, is not always the optimum from the point of view of either user welfare or social welfare. We would argue then that a regulatory authority would have strong argumentsi.e., welfare enhancement - to intervene by fixing a maximum value $b / W<1$ of leased spectrum. And these arguments are independent on the procedure that implements the bargaining on $p$.

\section{CONCLUSIONS}

The interaction between an MNO and an MVNO which compete for mobile users and which trade spectrum is analyzed in the paper. A three-level game has been developed. Bearing in mind the analysis of the results conducted in the previous section, we can conclude that:

1) Every actor, that is, users and the two operators, may be better off when the SO operator enters the market. For this to happen, the values $(b, p)$ should lay within the feasibility region.
2) The entry of the SO operator is only feasible if it improves the technology used by the incumbent operator.

3) The regulator intervention is deemed necessary in order to restrain the incumbent operator from leasing the whole amount of the spectrum to the entrant operator, which will harm the users.

\section{REFERENCES}

[1] M. Cave, C. Doyle, and W. Webb, Essentials of modern spectrum management, 2007.

[2] D. H. Shin and M. Bartolacci, "A study of MVNO diffusion and market structure in the EU, US, Hong Kong, and Singapore," Telematics and Informatics, vol. 24, pp. 86-100, 2007.

[3] J. Peha, "Sharing spectrum through spectrum policy reform and cognitive radio," Proceedings of the IEEE, vol. 97, no. 4, pp. 708-719, 2009.

[4] B. W. Kim and S. U. Park, "Determination of the optimal access charge for the mobile virtual network operator system," ETRI Journal, vol. 26, no. 6, pp. 665-668, Dec 2004.

[5] D. Fudenberg and J. Tirole, Game Theory. MIT Press, Cambridge, Massachusetts, 1991.

[6] H. Mutlu, M. Alanyali, and D. Starobinski, "Spot pricing of secondary spectrum access in wireless cellular networks," Networking, IEEE/ACM Transactions on, vol. 17, no. 6, pp. 1794 -1804, Dec. 2009.

[7] A. Al Daoud, M. Alanyali, and D. Starobinski, "Pricing strategies for spectrum lease in secondary markets," IEEE/ACM Transactions on Networking, vol. 18, no. 2, pp. 462-475, 2010.

[8] A. Banerjee and C. M. Dippon, "Voluntary relationships among mobile network operators and mobile virtual network operators: An economic explanation," Information Economics and Policy, vol. 21, pp. 72-84, 2009.

[9] R. Dewenter and J. Haucap, "Incentives to licence mobile virtual network operators (MVNOs)," in Access Pricing: Theory and Practice, Dewenter/Haucap, Ed. Amsterdam: Elsevier Science, 2006, pp. 305325.

[10] P. Kalmus and L. Wiethaus, "On the competitive effects of mobile virtual network operators," Telecommunications Policy, vol. 34, pp. 262-269, 2010.

[11] H. Le Cadre, M. Bouthou, and B. Tuffin, "A pricing model for a mobile network operator sharing limited resource with a mobile virtual network operator,' in Proc. of ICQT'09, LNCS 5539, 2009.

[12] — "Competition for subscribers between mobile operators sharing a limited resource," in Proceedings of Gamenets 09, Istanbul, Turkey, 2009.

[13] L. Guijarro, V. Pla, and J. Vidal, "Competition in cognitive radio networks: spectrum leasing and innovation," in Proc. of CCNC 2011, Jan. 2011.

[14] J. Wardrop, "Some theoretical aspects of road traffic research," Proc. of the Institute of Civil Engineers, vol. 1, pp. 325-378, 1952.

[15] E. Altman, T. Boulogne, R. El-Azouzi, T. Jimenez, and L. Wynter, "A survey on networking games in telecommunications," Computers \& Operations Research, vol. 33, no. 2, pp. 286-311, 2006.

[16] M. Katz and C. Shapiro, "Network externalities, competition, and compatibility," The American economic review, vol. 75, no. 3, pp. 424440, 1985.

[17] D. Niyato and E. Hossain, "Competitive pricing for spectrum sharing in cognitive radio networks: dynamic game, inefficiency of Nash equilibrium, and collusion," IEEE Journal on Selected Areas in Communications, vol. 26, no. 1, pp. 192-202, 2008.

[18] L. Guijarro and V. Pla, "Analysis of the competition between operators in a cognitive radio network scenario," Universitat Politècnica de València, Tech. Rep. TELPOL25c, 2010.

[19] A. Mukherjee and E. Pennings, "Tariffs, licensing and market structure," European Economic Review, vol. 50, no. 7, pp. 1699-1707, 2006.

[20] M. Osborne and A. Rubinstein, A course in game theory. The MIT press, 1994.

[21] P. Kalmus and L. Wiethaus, "On the Competitive Effects of Mobile Virtual Network Operators," Telecommunications Policy, vol. 34, pp. 262-269, 2010. 